This is a pre-copy-edited contribution of the following book chapter.

Matamala, Anna; Orero, Pilar (2019). "Training experts in inclusive practices for an equity on access to culture in Europe". S. Halder, V. Argyropoulos (eds) Inclusion, equity and access for individuals with disabilities. Palgrave Macmillan, 263-280.

\title{
Training Experts in Inclusive Practices for an Equity on Access to Culture in Europe
}

Anna Matamala and Pilar Orero

Universitat Autònoma de Barcelona, Spain

Access to cultural content should be offered by several services available by default. If access services are thought, and budgeted, in the production phase, they are better integrated, and cultural content can be enjoyed by all patrons. How to integrate access services in the production of any cultural good relays on education. Until accessibility enters the syllabus in primary and secondary education curricula -and also in higher education-, much works needs doing. Until such a time when accessibility is normalised as a must-carry requirement, the work of experts in media accessibility will be needed to add accessibility in postproduction stages. This is, in fact, the topic of this chapter: training in postproduction accessibility. The enterprise is looked from many angles, but in all cases putting the end user at the centre to understand their needs and expectations. To illustrate our approach to the subject, three European projects funded under the Erasmus + scheme will be used as examples: ACT (Accessible Culture and Training, http://pagines.uab.cat/act/), ADLAB PRO (https://adlabpro.wordpress.com/), and ILSA (Interlingual Live Subtitling for Access).

The chapter will begin with an introduction to the European legal context, which sets the research framework for both projects. Reference to related theories that guide our study will be made in this section. Training experts in accessibility in the scenic arts and defining their professional profile, which is the focus of the ACT project, will be discussed in Sections 2 and 3. The rationale of the project, the methodological tools used and the results obtained so far will be presented. Section 4 will define the rationale behind the ADLAB PRO and the ILSA project. The former aims to define the profile of the expert on audio description, an invisible access service with some EU regulation which needs to be increasingly fulfilled. The latter aims to define the profile of the professional live subtitler. Section 5 will then focus on ADLAB PRO and describe the methodological approach taken in the first part of project, the results, and the next steps to be taken. The article will conclude with some thoughts on the insights our research has put forward concerning the cultural barriers in Europe and the limitations persons with disabilities may find to attend cultural events, as well as the need for well-trained professionals at different levels: on the one hand, at the managing level and, on the other, at the actual provision of access services. A recommendation of mainstreaming accessibility as a default service to work towards a more equitable society will be proposed.

\section{Legal context in Europe and research framework}

It is important to know the legal situation in Europe regarding accessibility to understand the need for training experts in accessibility to cultural goods. The United Nations 
Convention on the Rights of Persons with Disabilities ${ }^{1}$ (CRPD) is the fundamental instrument and sets the context worldwide from where to develop accessibility plans and strategies. CRPD is legally binding and sets minimum standards for the rights of people with disabilities. It is the first human rights convention having the European Union as a party. The Council of the European Union (EU) adopted the Decision for the conclusion of the Convention ${ }^{2}$ on 26 November 2009. For the EU, the CRPD entered into force on 22 January 2011. This means that nowadays all the EU countries have signed the Convention, and only Ireland has not yet ratified it. There is also an Optional Protocol which out of the 27 EU countries 23 have also signed, and 21 have ratified $^{3}$. The CRPD aims to guarantee that people with disabilities can enjoy their rights on an equal basis with all other citizens, and it sets out minimum requirements for safeguarding a full range of civil, political, social, and economic rights. The convention reflects the EU commitment to building a barrier-free Europe for the estimated 80 million people with disabilities that will live in the EU in 2020, as set out in the European Commission disability strategy ${ }^{4}$. The plan outlines how the EU and national governments can empower people with disabilities so they can enjoy their rights. This shifts the study of accessibility from a clinical exclusive model -where, for instance, audio description was exclusively for the blind and subtitling was only for the deaf and hard of hearing- to a new inclusive for all paradigm. In fact, this new inclusive for all paradigm acknowledges that we all need accessibility. This is especially evident in a multilingual Europe, where $100 \%$ of EU residents require translation (or language accessibility) at one point.

An additional consideration to be made is that within the human rights framework there is a serious problem regarding accessibility, what Greco (2016) calls Accessibility as a Human Right Divide Problem (AHRD Problem). The AHRD Problem highlights the issue between accessibility as a human right per se versus accessibility as an instrument for the fulfilment of human rights. It is in this latter proactive principle where media accessibility is framed in this chapter: access as a necessary requirement for achieving human rights.

Europe has responded to the obligations derived from the UNCRPD with two important EU legislations, establishing the context for the development of professional experts in accessibility: the EU directive known as Audiovisual Media Service Directive (AVMSD) and the European Accessibility Act (EAA). The EU directive ${ }^{5}$ 2010/13/EU in Article 46 explicitly acknowledges that the "right of persons with a disability and of the elderly to participate and be integrated in the social and cultural life of the Union is inextricably linked to the provision of accessible audiovisual media services. The means to achieve accessibility should include, but need not be limited to, sign language, subtitling, audio-description". The European Accessibility Act ${ }^{6}$ (EAA) aims "to improve the functioning of the internal market for accessible products and services by removing barriers created by divergent legislation".

These legislations will allow for a full integration in cultural life of all citizens. The proportion of people in need of accessibility is increasing in the EU, partly due to an increasing ageing population, but also to the mobility of its citizens, who for work or tourism change their country and move to a new location and a new language. Accessibility has

\footnotetext{
${ }^{1}$ https://www.un.org/development/desa/disabilities/convention-on-the-rights-of-persons-withdisabilities.html (consulted on 02-08-2017)

${ }^{2}$ http://eur-lex.europa.eu/legal-content/EN/TXT/PDF/?uri=CELEX:32010D0048\&rid=1 (consulted on 02-08-2017)

${ }^{3} \mathrm{http} / / /$ europa.eu/rapid/press-release IP-11-4_en.htm (consulted on 02-08-2017)

4 http://europa.eu/rapid/press-release IP-10-1505 en.htm?locale=en (consulted on 02-08-2017)

${ }^{5}$ Directive 2010/13/EU of the European Parliament and of the Council of 10 March 2010 on the coordination of certain provisions laid down by law, regulation or administrative action in Member States concerning the provision of audiovisual media services (Audiovisual Media Services Directive) (http://eur-lex.europa.eu/legal-content/EN/ALL/?uri=CELEX\%3A32010L0013) (consulted on 02-082017)

${ }^{6}$ European Accessibility Act (http://ec.europa.eu/social/main.jsp?catId=1202) (consulted on 02-08-2017)
} 
long been a critical issue in the EU culture sector as part of the Digital Single Market $^{7}$. A Digital Single Market (DSM) is one in which the free movement of persons, services and capital is ensured and where the individuals and businesses can seamlessly access and exercise online activities under conditions of fair competition, with a high level of consumer and personal data protection, irrespective of their nationality or place of residence. On 10 May 2017, the Commission published the mid-term review of the Digital Single Market Strategy. It shows the progress made in implementing the Strategy since 2015 and where further actions are needed ${ }^{8}$. However, no much attention has been paid to cultural representations such as the scenic arts beyond the introduction of translation services such as surtitling in opera (Orero, 2007; Matamala \& Orero, 2013). Access services are often not implemented due to lack of awareness on what is needed in terms of technology, and also on how to contract those who produce them. Therefore, training experts in these specific fields is much needed in the current European context.

\section{Training on accessibility to the scenic arts: rationale and framework}

The time has come to establish the new professional profile of accessibility manager, and its training, for the specific environment of scenic arts and live events. Most opera houses in Europe offer subtitles or surtitles (Oncins, 2015), and other access services such as audio description (Matamala \& Orero, 2014; Oncins et al., 2013; Weaver, 2011), sign language interpreting or touch tours are offered in some playhouses too (Eardly-Weaver, 2013). However, their provision is uneven (ADLAB, 2014) and finding out the access services on offer for a specific performance may prove challenging. This applies to many cultural goods, be it in museums, cinemas, opera or play houses.

With the must-carry access requirements established in the EAA, some national governments have started to make provisions by developing an active accessibility policy. This is the case for the Catalan Government through its Department of Culture, where the new Catalan Accessibility Code sets some requirements from the Accessibility Decree ${ }^{9}$. Other countries such as Germany, in Baviera, and Sweden have also started working towards setting up accessibility requirements for publicly funded cultural productions.

Looking at one case, the Catalan, clear instructions have been issued to both private and public exhibitors: they must draft an accessibility plan that considers not only physical accessibility and health and safety regulations but also provides a joint territorial planning of access services, a minimum percentage of performances offered with audio description, and a minimum percentage offered with subtitles. The protocol establishes that the provision of subtitles and audio descriptions does not have to be offered necessarily at the same time, and that clear information should be provided about the access services on offer. The accessibility plan is expected to be revised every four years, in order to improve the number of accessible performances, and the Catalan Department of Culture will be the body responsible for checking that all requirements are fulfilled.

As part of the plan to implement the Catalan Accessibility Code, some actions were prepared. The first was to find out the state of the art regarding accessibility in the $30+$ public playhouses in Catalonia. After elaborating and sending a questionnaire, it was clear that accessibility actions, if any, were shared by different departments within one institution and that a department of accessibility centralising all access services was seldom found. There was no person responsible for all accessibility actions, and often one department did not liaise with another. So, for instance, the person selling tickets would not be familiar with sitting arrangements for wheelchairs, nor with the provision of signed performances. Therefore, one direct outcome from this research was the need to centralise accessibility in one department, with a person responsible for all issues. This person, the accessibility

\footnotetext{
${ }^{7}$ http://eur-lex.europa.eu/legal-content/EN/TXT/?qid=1447773803386\&uri=CELEX\%3A52015DC0192 (consulted on 03-08-2017)

8 https://ec.europa.eu/digital-single-market/en/news/contributions-and-preliminary-trends-publicconsultation-audiovisual-media-services-avmsd (consulted on 03-08-2017)

${ }^{9}$ http://portaldogc.gencat.cat/utilsEADOP/PDF/6742/1379017.pdf (consulted on 02-08-2017)
} 
expert for the scenic arts, or even for the broader field of culture management, is not described in any of the official professions in Eurostat ${ }^{10}$ but it may exist in other places away from Europe where a more stringent legislation has been in place for years, such as the USA. Accessibility management exists in other areas of accessibility such as the Web or the field of physical accessibility in urban development ${ }^{11}$, transport $^{12}$ or public administration ${ }^{13}$.

The need to define the professional profile of the accessibility expert in the scenic arts, with their skills and competences, was therefore confirmed through this initial research. Since EAA and AVMSD are at EU level, and the aim was to work within the Single Digital Market framework, it was deemed necessary to define the new professional profile beyond Catalonia and across Europe. For this reason, a EU action was started taking advantage of the funding available from the Erasmus+ programme ${ }^{14}$. This programme aims to support the potential of Europe's talent and social assets in a lifelong learning perspective, including support to formal, non-formal and informal learning. More specifically, a joint EU project -Accessible Culture Training (ACT $)^{15}$ - was submitted and funded. ACT is transnational, a basic requirement when upscaling at pan-European level, because not only access services have to be considered but also the cultures and languages in which they will be implemented. In Europe, there is a wealth of language situations (bilingual, monolingual, multilingual) and translation traditions (subtitling, dubbing and voice-over), where cultural representations will take place.

To design a truly useful proposal, it was considered important to count with a bottom-up transectorial collaboration. ACT gathered as partners key stakeholders in the media accessibility field: training institutions, governmental authorities, theatre managers, certification organisation, and end users. Four universities (Universitat Autònoma de Barcelona, University of Antwerp, Queen's University Belfast, and University of Vienna), two theatres (Transit, in Spain, and NTGEnt, in Belgium), two governmental departments (Inter, a technical partner of the Flemish government, and the Department of Culture from the Catalan government), and a EU quality certification agency (ECQA). ECQA was asked to participate to secure resilience and to organise tests for certification beyond university courses. Especially challenging is the certification approach towards informal learning proposals, something alien to universities. Finally, end users from all countries participated in the project through associations as associate partners.

\section{Profiling the expert in accessibility for the scenic arts: methodological aspects and results}

The project ACT follows a bottom-up methodology, gathering information from users within the UN CRPD framework, where "nothing for us without us" is defined as a departing point for any action. Partners in the project identified three groups to offer feedback on their accessibility experience and expectations: art venues managers, user associations, and artistic teams (Orero, forthcoming). To gather feedback a survey was drafted, pre-piloted and then translated into the languages of the countries in the project (Catalan, Dutch, English, and German). Data from venues were collected through this survey, and

\footnotetext{
${ }^{10} \mathrm{http}$ ://ec.europa.eu/eurostat/statistics-explained/index.php/Europe 2020 indicators_-_employment (consulted on 02/08/2017)

${ }^{11} \mathrm{http}: / / w w w . b e n d b u l l e t i n . c o m / n e w s / 1481864-151 /$ bend-names-duncan-new-accessibility-manager (consulted on 02/08/2017)

12 https://www.reed.co.uk/jobs/schools-liaison-and-accessibility-manager/32388489 (consulted on 02/08/2017)
}

http://agency.governmentjobs.com/bend/default.cfm?action=viewclassspec\&classSpecID $=888377 \&$ agenc $\mathrm{y}=2242 \&$ viewOnly=yes (consulted on $02 / 08 / 2017$ )

${ }^{14} \mathrm{http}$ ://ec.europa.eu/programmes/erasmus-plus/resources en (consulted on 02/04/2017)

${ }^{15} \mathrm{http}: / /$ pagines.uab.cat/act/ (consulted on 02/08/2017) 
complemented by focus groups with artistic teams and individual interviews with end users. The questions asked for the venues were:

(1) to what extent are the various access facilities (e.g. audio description, easy-toread materials, sign language interpreting, audio subtitling) offered in the different arts venues in each partner country and how does this vary?

(2) how are access facilities promoted?

(3) who is responsible for accessibility?

(4) what access quality control measures are in place?

(5) what type of accessibility training is offered if any?

(6) what challenges are faced by venues regarding accessibility?

Results from the three interventions led to some interesting conclusions. When the venue managers replied, it seemed that they all took into consideration accessibility and they complied to a very high degree with accessibility requirements. This was surprising until it was clarified what accessibility requirements meant for them: physical accessibility. Nowadays it is almost impossible to get a planning permission to open a public venue unless basic measures are met: accessible toilets, ramps as alternative for steps, lifts for motorised wheelchairs, and attention to health and safety regulations. Venue managers considered they met requirements and this clashed with results from the comments by the end users, who had further needs from the basic adapted toilet and ramps. Finally, a third group of informants was artists. They were added hoping to gather some feedback from any artist who had considered accessibility in the production of their art. This proved to be an awareness exercise, since artists considered artistic accessibility as the inclusion of persons with disabilities as actors in the regular performances, or as the creation of specific shows exclusively for persons with disabilities.

Raising awareness on media accessibility was again the priority surfacing the study. Informing on media access services and possibilities to all three groups was a necessary step and some actions were taken. The research group TransMedia Catalonia, leader of the ACT project, decided to create some short videos to illustrate issues such as: how to reach the venue and how to communicate access services through the website ${ }^{16}$ or seating distribution ${ }^{17}$, to name two. The Catalan Department of Culture, also partner in ACT, decided to organise three workshops addressing: exhibitors, end users, and users of accessible technologies. The workshops aimed at:

a. Understanding the characteristics and the specific needs of people with disabilities,

b. Consider these groups when programming activities,

c. Gain experience through practical examples, and

d. Identify barriers to access and design their corresponding solutions.

Videos and recording ${ }^{18}$ of the workshops are available online, hoping to fulfil basic information when dealing with media accessibility.

The feed-back gathered from different group of informants was also used to draft a list of requirements (Eardley-Weaver et al., forthcoming), and the next step was to define the expert's skills and competences and also its denomination departing from this input. Two different but complementary profiles appeared: The Accessibility Coordinator, and the Accessibility Manager. The former works at a venue and takes care of all accessibility needs for successful integration of all people to access culture. Meanwhile, the latter, the Accessibility Manager, oversees cultural event management with a focus on accessibility and is not necessarily based in any venue, such as a theatre. An accessibility manager may be in charge of coordinating accessibility at various venues within a local district. This work will involve collaborating with accessibility coordinators at individual venues where such a role exists. If there is no accessibility coordinator for any given individual venues

\footnotetext{
16 https://youtu.be/-fns2FsJWC8 (consulted 02/08/2017)

17 https://youtu.be/hgcCpKaX jo (consulted 02/08/2017)

18 https://sde.cultura.gencat.cat/cultura/curs-d'accessibilitat-als-espais-escenics-imusicals_adh_1574.html (consulted 02/08/2017)
} 
(such as in smaller venues), it may be required from the accessibility manager to oversee accessibility across these venues. Both profiles are experts on accessibility and should be able to decide accessibility policies or adequacy when choosing venues or productions.

The two job roles share many traits in the basic skills definitions such as: understanding accessibility, venue accessibility, access services, accessibility management for live events, and promoting accessibility. The main distinction between the two functions resides in the context the two specialists operate. The accessibility manager must, in fact, have an additional skill: be flexible and be able to transfer his knowledge and competences to different environments. This applies to physical skills (i.e. related to the venue) and to interpersonal skills.

\section{Defining the audio describer and live subtitler professional profiles}

The ACT project takes a broader look to accessibility, focusing on the profile of those coordinating or managing the accessibility in one or various venues. It does not look at the specific training those providing access services should have. However, this is a topic that merits further research as the professionals providing access services such as audio description or live subtitling vary in their profile and training across European countries.

In the area of subtitling, a key priority for the users is to access live content such as news and public events. Live subtitling through speech recognition, known as respeaking, has consolidated as the preferred method to provide live subtitles around the world (Romero-Fresco, forthcoming a). Respeakers listen to the soundtrack of a programme and simultaneously repeat and rephrase what they hear to a speech recognition software that turns these utterances into subtitles (Romero-Fresco, forthcoming b). Although this method is very successful for same language subtitles, a new challenge has emerged (RomeroFresco \& Pöchhacker, forthcoming). Migration streams and the increased multilingual composition of societies have led to a growing demand for access to live content in a foreign language. Same language live subtitlers and interlingual live subtitlers produced by respeaking are in urgent need to be developed as professional profiles. The need to train quality professionals in these techniques is now a priority, thus providing access to live content not only for deaf people but also for foreign audiences, including migrants and

refugees. The Interlingual Live Subtitling for Access (ILSA) project (http://www.ilsaproject.eu) aims to design, develop, test, and produce a protocol for the implementation of subtitling in four real-life scenarios: TV, conferences, the classroom, and the Parliament. The curriculum and training materials developed by the project will be flexible. This means they can be integrated in different learning environments from higher education students to professionals already working in translation and accessibility. The ILSA consortium includes four universities (Vigo University, Antwerp University, University of Warsaw and University of Vienna) and three non-academic partners: the Galician Parliament, the public Belgium TV broadcaster VRT and Dostepni.eu, the first company to produce intralingual live subtiles for TV and social events in Poland.

Concerning audio description (AD), it is a service where accessibility is achieved through the insertion of short verbal descriptions illustrating the essential visual elements of an audiovisual product, mainly for the blind and visually impaired community. The need to improve the practice of audio description and to make more and more audiovisual products available to sensorially disabled people is ever more urgent due to the significant number of people in Europe registered as blind or described as having low vision, or the elderly. Inconsistencies in $A D$ provision and crafting methods were identified by ADLAB, (http://www.adlabproject.eu/), a project that produced the first reliable and consistent European guidelines for the practice of audio description (Remael et al., 2015). Based on the results of this project, a new project (ADLAB PRO) was submitted and funded. The project aims to create free-access flexible didactic materials that will contribute to train a still rather unknown professional figure, that of the audio describer. The project, led by University of Trieste, gathers partners from academic institutions (Universitat Autònoma de Barcelona, University of Trieste, Adam Mickiewicz University), broadcasters (Slovenian Television), SMEs involved in the production of access services (Utopian Voices, Soundfocus), and end user's associations (RNIB). 
Only through research-informed comprehensive training programmes such as the ones proposed by ACT, ADLAB PRO and ILSA will it be possible to ensure that these new professional profiles on media accessibility meet the required standards regarding the product and the working conditions. This is an essential step to guarantee a truly wider access that can include and integrate all citizens in the audiovisual, educational, political and social life of the countries in which they are living.

\section{Mapping audio description training in Europe: methodology and preliminary results in ADLAB PRO}

The first necessary step within the ADLAB PRO project was to identify current audio description training practices across Europe. To this end, two methodological approaches were taken: a quantitative and a qualitative analysis. The former was based on a questionnaire distributed to $A D$ trainers in academic and non-academic institutions in Belgium, Italy, the Netherlands, Slovenia, Spain, the UK and Poland, among others. The qualitative analysis was performed on selected course materials provided by $A D$ trainers from the partner institutions.

The online questionnaire gathered data about the quantity and length of the audio description training, the types of training offered, the group sizes, the teaching modes, the amount of theory and practice in the courses, as well as the assessment modes implemented. Special emphasis was put into the competences, skills and learning outcomes expected to be achieved by learners when completing the courses. 86 respondents completed the survey, giving information about a total of 192 courses across Europe (93 academic and 99 non-academic), and results, as reported by Chmiel and Mazur (2017), seem to indicate that the majority of AD teaching concerns film audio description. When asked about the importance of specific competences in AD training on a 5-point Likert scale, "choosing the most relevant information to describe" (4.87) and "perfect use of mother tongue" (4.70) ranked first, followed by "knowledge of the needs of the visually impaired" (4.65), "choosing appropriate AD strategies" (4.46), "technical aspects (editing, timing, text compression) (4.32), "knowledge of cinematography, theatre, arts, and/or semiotics of the image (3.84), "vocal skills" (3.52), "IT-related skills" (3.51) and "reflecting filmic language" (3.22). Concerning transferrable skills, "efficient work organisation and time management" (4.45), "ethics" (4.29), "self-development" (4.15) and "teamwork" were all considered of high importance.

The questionnaire also shed light on the main features of $A D$ courses: both academic and non-academic are generally under 20 hours long, and are generally addressed to larger groups in academic environments (10-20 and more, in contrast with groups under 10 participants or medium-sized groups below 20 in non-academic environments). Academic courses are generally taught at MA level, generally within courses in audiovisual translation. Most courses rely on traditional face-to-face teaching, and only some of them offer e-learning or blended learning. In terms of skills, it seems that there are no major differences except for the fact that non-academic courses tend to focus more on voicing and recording than their academic counterparts. Concerning contents, academic courses tend to include more presentations of theoretical concepts, based on research articles and projects, as compared to non-academic contexts. However, no statistical significant differences between the two types of courses were observed. Both type were also found to be mainly based on practical exercises, analysis of existing audio descriptions, and discussion of guidelines. It is also interesting to notice that most trainers have experience both in teaching and in AD creation.

Regarding the qualitative analyses of courses (see Chmiel \& Mazur, 2017, for further details), two long academic courses, one medium-length academic course, one long non-academic course and one short non-academic course were chosen as case studies. They all show different features in terms of length and modality (online/blended/in class), but they all combine teacher-centered with student-centered learning activities and rely extensively on the use of audiovisual content. Analysing existing audio descriptions, discussing audio description guidelines, presenting theoretical aspects, and doing practical exercises are the main activities in all cases. Overall, it seems that, despite differences in 
approaches and specific features, there is common ground among the different academic and non-academic courses.

Departing from this mapping of the audio description training situation across Europe, a second step has been taken. An online questionnaire has been designed and circulated extensively to audio description providers, audio describers and end-users to gather feed-back on the audio describer profile. More specifically, the questionnaire aims to collect information on the audio description profile in terms of previous training and experience, stages in which the audio describer is involved, working conditions, and cooperation with persons with disabilities. It also aims to elucidate how professionals view their work, the difficulties they encounter, and the skills and competences that are considered more relevant. The voice of the end user is also expected to be heard with specific questions concerning their use of audio description in different environments, and their views on the job of the audio describer. The questionnaire also includes question about user satisfaction and the aspects user consider most important in quality audio description. The analysis of this second questionnaire is currently ongoing, but it is expected to shed light on the skills and competences an audio describer should acquire.

Based on this list of skills and competences, the next steps will include the design of a course and the development of course contents, which will need to be evaluated and tested in terms of quality and attributed credits.

\section{Conclusions: towards mainstreaming accessibility}

The European Union has taken remarkable steps towards accessibility and inclusion. The first is the exceptional signature -as a single party- of the first human rights convention by the Council of the European Union (EU $)^{19}$ on 26 November 2009. Since that date an active policy has been developed along a generous funding both for research in the field of media accessibility and its training. Europe as a region, with its rich diversity in cultures and languages, needs to secure the interaction of citizens beyond geopolitical areas. The adoption of positive inclusive policies towards all EU citizens shows the understanding towards diversity and the need to address the different needs in a unified way. By understanding the requests and expectations of the most vulnerable sectors in society, specific actions have been activated. Europe views accessibility for persons with disabilities, but also recognises the needs for accessibility for the largest population in Europe: the aged. Europe has also been subject of migrations within EU countries, and also from other neighbouring places. This is the specific objective of the societal challenge 'Europe in a changing world - Inclusive, innovative and reflective societies' funded to foster a greater understanding of Europe, provide solutions, and support inclusive, innovative and reflective European societies in a context of unprecedented transformations and growing global interdependencies.

The integration of the displaced citizens -who in turn can also be persons with disabilities- is also considered by Europe as a priority in need of dynamic solutions. Pressure from migration flows, socio-economic and cultural changes due to new forms of human-technology interaction under the fourth industrial revolution can impact the future of Europe at many levels. Until accessibility becomes normalised and integrated in syllabus across disciplines and education levels, until accessibility is mainstreamed in a more equitable society, a special effort has to be made in training those responsible for providing it as a postproduction service. The quality and diversity of services has to be understood so access is offered to the widest possible diversity of user profiles thus promoting inclusion and equity on access to culture and education in Europe.

\section{Acknowledgements}

This chapter is part of the ACT (2015-ES01-KA2013-015734) and ADLAB PRO (2016-1IT02-KA203-02431) projects, supported by the Erasmus + programme of the European Union. The content of this chapter does not reflect the official opinions of the European

\footnotetext{
${ }^{19}$ http://eur-lex.europa.eu/legal-content/EN/TXT/PDF/?uri=CELEX:32010D0048\&rid=1 (consulted on 02-08-2017)
} 
Union. Responsibility for the information and views expressed in this chapter lies entirely with the authors. The authors are part of TransMedia Catalonia research group, funded by the Catalan Government through the SGR funding scheme.

\section{References}

Chmiel, A. \& Mazur, I. (2017). Assessment of current AD training practices. ADLAB PRO. Retrieved from https://adlabpro.files.wordpress.com/2017/07/20170608_uam_io1_report.pdf

Eardly-Weaver, S. (2013). Opening eyes to opera: The process of translation for blind and partially-sighted audiences. Translation and Interpreting Studies. The Journal of the American Translation and Interpreting Studies Association, 8(2), 272 -292.

Eardley-Weaver, S., Eardley, T., Remael, A., Robert, I., Matamala, A. \& Orero, P. (forthcoming). ACT now: Cultural Accessibility Training and Profiling.

Greco, G. M. (2016). On Accessibility as a Human Right, with an application to Media Accessibility. In A. Matamala \&P. Orero (Eds.), Researching Audio Description (pp. 11-33). London: Palgrave Macmillan.

Matamala A. \& Orero, P. (2013). Audiovisual Translation, When modalities merge. Perspectives. Studies in Translatology, 23 (1), 2-4.

Matamala, A. \& Orero, P. (2014). Formar en accessibilitat als mitjans: noves necessitats socials, noves tecnologies, noves propostes dinàmiques. Revista CIDUI, 2, 1-8.

Oncins, E. (2015). The tyranny of the tool: surtitling live performances. Perspectives.

Studies in Translatology, 23(1), 42-61.

Oncins E., Lopes, O., Orero, P. \& Serrano, J. (2013). All Together Now: A multi-language and multi-system mobile application to make live performing arts accessible. JosTrans, 20, 147-164.

Orero, P. (2007). Audiosubtitling: A Possible Solution for Opera Accessibility in Catalonia. TradTerm, 13, 135-149.

Orero, P. (forthcoming). The professional profile of the expert in media accessibility for the scenic arts. Rivista internazionale di tecnica della traduzione RIIT.

Remael, A., Reviers, R. \& Vercauteren, G. (2015). Pictures Painted into Words: ADLAB Audio Description Guidelines. Edizioni Università di Trieste: Trieste.

Romero-Fresco, P \& Pöchhacker, F. (forthcoming). Quality assessment in interlingual live subtitling: The NTR model. Linguistica Antverpiensia, New Series: Themes in Translation Studies, 14.

Romero-Fresco, P. (forthcoming a) Respeaking: subtitling through speech recognition. In L. Pérez-González (Ed.), The Routledge Handbook of Audiovisual Translation Studies.

London/New York: Routledge.

Romero-Fresco, Pablo (forthcoming b). Reception Studies in Live and Pre-Recorded SDH. In E. Di Giovanni and Y. Gambier (Eds.), Reception Studies and Audiovisual Translation. Amsterdam: John Benjamins. 
Weaver, S. (2011). Opening doors to opera. The strategies, challenges and general role of the translator. InTralinea 12. Retrieved from http://www.intralinea.org/archive/article/1660 